\section{FT-ICR Reaction Experiments and Molecular Dynamics Simulations of Precursor Clusters for SWNTs}

S. Maruyama, M. Kohno, T. Mukae, Y. Shibuta and $\mathrm{S}$. Inoue

Engineering Research Institute and

Department of Mechanical Engineering, The University of Tokyo

2-11-16 Yayoi, Bunkyo-ku, Tokyo 113-8656, Japan

Generation mechanism of single walled carbon nanotubes is investigated through experimental and molecular dynamics simulation studies of interaction of metal atom and carbon clusters. Fourier Transform Ion Cyclotron Resonance (FT-ICR) mass spectrometer directly connected to the laser-vaporization cluster beam source (1) was used for the chemical reaction experiments of metal-carbon binary clusters generated by the laservaporization of $\mathrm{Ni} / \mathrm{Co}$ or $\mathrm{Ni} / \mathrm{Y}$ loaded carbon materials used for macroscopic production of SWNTs. Positive and negative mass spectra of clusters for $\mathrm{Ni} / \mathrm{Co}(0.6$ at $\% \mathrm{Ni}$ and $0.6 \%$ Co) loaded sample are shown in Fig. 1. There was not a trace of $\mathrm{Ni}$ or $\mathrm{Co}$ in the positive mass spectrum and tiny signals of $\mathrm{NiC}_{\mathrm{n}}$ and $\mathrm{CoC}_{\mathrm{n}}$ were measured for negative spectrum as in Fig. 2(a) (expanded view of Fig. 1(b)). More drastic effect of doping of $\mathrm{Ni} / \mathrm{Co}$ was observed in pure carbon cluster distribution such as the prominent $\mathrm{C}_{60}{ }^{+}$and $\mathrm{C}_{70}{ }^{+}$in positive spectrum (Fig. 1(a)) and even-odd intensity alternations in $\mathrm{C}_{30}{ }^{-}$through $\mathrm{C}_{60}{ }^{-}$ range in negative spectrum (Fig. 1(b)). Both suggest the rapid growth of caged carbon clusters. The chemical reaction of these clusters with $\mathrm{NO}$ was used as the probe of the structure of clusters. The reaction results with $10^{-7}$ Torr NO for $2 \mathrm{~s}$ and $10 \mathrm{~s}$ are shown in Fig. 2(b) and Fig. 2(c), respectively. The chemisorption of $\mathrm{NO}$ to $\mathrm{NiC}_{n}{ }^{-}$and $\mathrm{CoC}_{\mathrm{n}}{ }^{-}$were observed in much faster rate than pure carbon clusters and La-carbon binary clusters (1). The high reactivity of $\mathrm{NiC}_{\mathrm{n}}{ }^{-}$and $\mathrm{CoC}_{\mathrm{n}}{ }^{-}$strongly suggests that $\mathrm{Ni}$ or Co atom is outside the carbon cage. Similar chemical reaction experiments for the Ni-Y loaded sample showed that $\mathrm{Ni}$ atom was again outside the carbon cage but $\mathrm{Y}$ atom was inside the carbon cage just-like the precursor clusters to the endohedral metallofullerene (1).

The formation process of metal-carbon binary clusters was also studied using the molecular dynamics simulations. The growth process of carbon clusters from completely random vapor phase was simulated with and without $1 \%$ of metal atoms (2). Inclusion of La atoms enhanced the clustering process to the random caged carbon clusters, with a La atom inside the cage. However, a $\mathrm{Ni}$ atom on the face of the random cage (Fig. 3(a)) prohibited the complete anneal of the cage structure. On the other hand, it was found that collisions of such imperfect random-cage clusters lead to the elongated giant fullerene (Fig. 3(b)) with the similar diameter as $(10,10)$ SWNT. It is speculated that the abundant generation of imperfect random-cage carbon clusters by a metal atom and collision product of such clusters are the initial seed of gas phase growth of SWNTs.

Part of this work was supported by Grant-in-Aid for Scientific Research (B) (No. 12450082) from the Ministry of Education, Science, Sports and Culture, Japan.

\section{REFERENCES}

1. S. Maruyama et al., Fullerene 2000, ECS, submitted.

2. Y. Yamaguchi and S. Maruyama, Euro. Phys. J. D, 9, 1-4, 385 (1999).

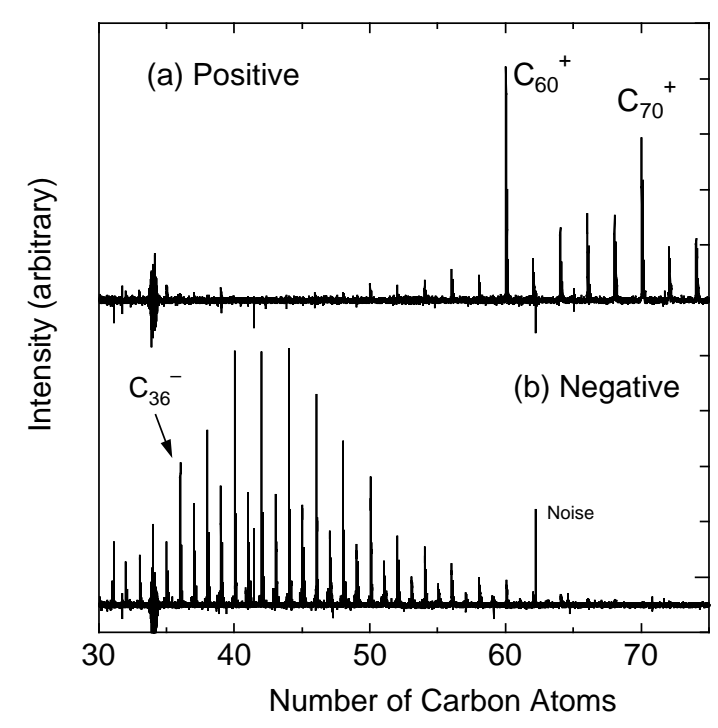

Fig. 1 As injected positive and negative clusters from $\mathrm{Ni} / \mathrm{Co}$ loaded carbon sample.

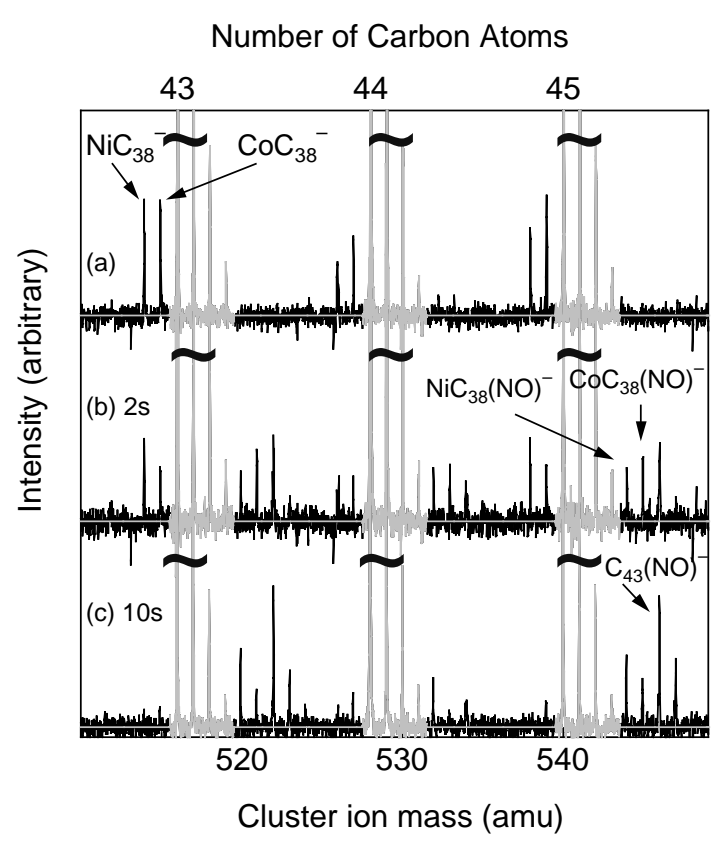

Fig. 2 Reaction of $\mathrm{NiC}_{38}{ }^{-}$and $\mathrm{CoC}_{38}{ }^{-}$with $\mathrm{NO}$. Note that signal of pure carbon are drawn in gray lines.

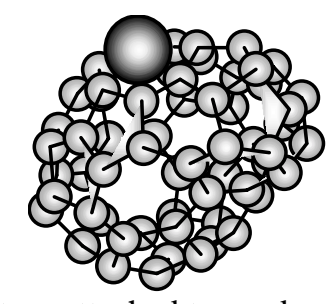

(a) $\mathrm{Ni}$ atom attached to random-cage $\mathrm{C}_{60}$

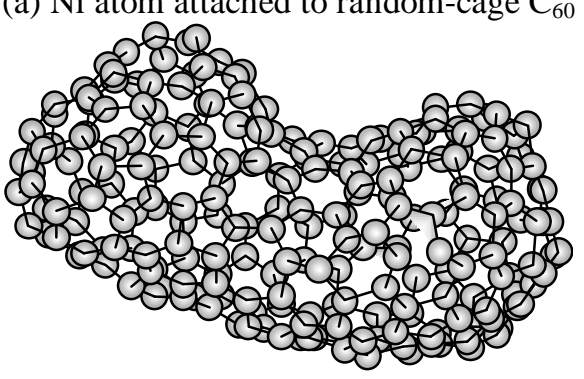

(b) Elongated giant fullerene

Fig. 3 Snapshots of structures obtained with molecular dynamics simulations. 